\title{
Algoritmo para el diseño de base de datos.
}

\section{Algorithm for database design.}

Vanessa Lorena Valverde González. ${ }^{1}$

Recibido: 10-05-2019 / Revisado: 15-06-2019 /Aceptado: 04-07-2019/ Publicado: 28-07-2019

\begin{abstract}
.
DOI: https://doi.org/10.33262/cienciadigital.v3i3.2.1.778

The present work is a proposal to direct the design of Databases, without taking into account the programming language or the Database Management System (SGBD) to be used by the programmer / developer. The intention of it is to know about Database applied to computing, choose two design models and implement them in a methodology that has software engineering objective and applied to the design of the database, which in itself is the center of the development application. An algorithm with a methodology, that optimizes the time to creation of a database, being a sequence of steps, an orderly process is facilitated through logic. The algorithm has been evaluated in a complexity $\mathrm{O}(1)$, that it is efficient because if the data increases or there are changes in them, the algorithm will be offering results in a finite process without any difficulty.Time New Roman 12 line spacing 1.15 (150 - 300 words).
\end{abstract}

Keywords: Algorithm, Database, Design, Entity Relationship.

\section{Resumen.}

El presente trabajo es una propuesta para direccionar el diseño de Bases de Datos, sin tomar en cuenta el lenguaje de programación o el Sistema Gestor de Base de Datos (SGBD) a utilizar por el programador / desarrollador. La intención del mismo es conocer acerca de Base de Datos aplicadas a la informática, escoger dos modelos de diseño e implementarlos en una metodología que tenga como objetivo la ingeniería del software, aplicado al diseño de la base de datos, que en si es el centro del desarrollo de

1 Escuela Superior Politécnica de Chimborazo. Facultad de Mecánica, Riobamba, Ecuador, v_valverde@espoch.edu.ec 
una aplicación, un algoritmo con una metodología que optimiza el tiempo para la creación de una base de datos, al ser una secuencia de pasos se facilita de manera ordenada un proceso a través de la lógica. El algoritmo ha sido evaluado en una complejidad O (1). Lo que resulta eficiente, por más que aumente los datos o exista cambios en los mismos el algoritmo cumplirá con ofrecer resultados en un proceso finito sin dificultad alguna.

Palabras claves: Algoritmo, Base de Datos, Diseño, Entidad Relación.

\section{Introducción.}

Hoy en día toda actividad que se realice en las tareas cotidianas se las desea informatizar, al estar involucrados en la era digital, objeto real o abstracto no está fuera del alcance de la informática. La digitalización de Datos en las Bases no es nuevo, pero en la actualidad existe una ingeniería del software enfocado al diseño de las mismas es por esto que se plantea a través de un algoritmo (secuencia de pasos ordenada el desarrollo de una metodología que ayude a los desarrolladores de software a mejorar lo tiempos en el desarrollo de software con Bases de Datos); la Ingeniería de Software ayuda a la creación de un software desde los requerimientos de usuario hasta su producción definitiva, para la presente investigación lo que se realiza es la Ingeniería del Software enfocada sólo al área de diseño de base de datos.

El desarrollar un algoritmo para la creación de una base de datos implica los siguientes objetivos: definir conceptos de bases de datos y algoritmos, elaborar el algoritmo que facilite la aplicación de una metodología realizada a partir de las fases de diseño de bases de datos.

Rivas et al. (2015). Afirman que "El concepto de metodología es "conjunto de métodos coherentes y relacionados por unos principios comunes". La fase metodología será conocer los modelos y fases de diseño de una Base de Datos a utilizar en el desarrollo de la investigación, plantear el algoritmo y analizar su nivel de complejidad.

El interés de proponer un algoritmo que facilite el diseño de una Base de Datos es para quien se interese en el tema de las Bases de Datos conozca un forma lógica y fácil de realizar en proceso, siempre el trabajar de forma ordenada optimizará tiempos en los trabajos a realizar, además servirá en la aplicación de la docencia, así como en la vida cotidiana de un programador/desarrollador.

\section{Metodologia.}

Una metodología no intenta resolver un problema lo que intenta es tratar de cometer la menor cantidad de errores posibles y tratar de cumplir tiempos a través de su planificación implementado modelos.

Codina. (2017). Describe que "una base de datos es un sistema que debe ser capaz de satisfacer las necesidades de información de sus usuarios, relacionando preguntas con 
documentos". La Ingeniería del Software analiza el ciclo de vida de un sistema a raíz del concepto general lo que se desarrollará es el ciclo de vida enfocado al diseño de una Base de Datos a través de la comparación de diferentes metodologías.

El concepto de Ingeniería del Software surge de la distinción entre el desarrollo de pequeños proyectos (programming in the small) y el desarrollo de grandes proyectos (programming in the large), de forma que el reconocimiento de que la Ingeniería del Software está relacionada con esta última. (García, 2018)

Por lo tanto, el desarrollo del ciclo de diseño de Base de Datos también está relacionado con el desarrollo de Bases de Datos pequeñas y grandes Bases de Datos. Una Base de Datos en términos informáticos es una colección de datos inter relacionados almacenados en un repositorio tecnológico en el cuál se pude almacenar grandes cantidades de información las cuales pueden ser manipuladas por el usuario según las facilidades del sistema que acceda a la misma. Sólo a través de un gestor de base de datos se puede tener acceso a dichos datos.

Una base de datos es una colección de datos interrelacionados y un sistema de gestión de bases de datos SGBD (DBMS por sus siglas en inglés DataBase Management System), es el software que gestiona y controla el acceso a los datos de la base de datos. Una aplicación de base de datos es un programa que interactúa con la base de datos. (Merchán, 2016)

La metodología es un conjunto de métodos que ayudan a predecir el éxito de un Sistema, mientras que un modelo es una serie de pasos a seguir para llegar a un fin de forma planificada, es decir pasos, reglas y procedimientos para llevar a cabo la solución de un problema; por otro lado, la metodología realiza el análisis de las fortalezas y debilidades a raíz de los modelos, recordando que no existe un método mejor que otro, todo dependerá del sistema de base de datos a implementar.

La existencia de modelos conceptuales existentes de objetos y fenómenos, especificando que en los años setenta del siglo XIX fue ideado un modelo con el propósito de simplificar el diseño de una base de datos, a través de diagramas de entidad-relación. Se hace la orientación del objetivo de representar la estructura lógica de una base de datas en forma de gráfico. Se determina los elementos esenciales del diseño de una base de datos, referente a las entidades, atributos o campos y relaciones entre entidades. (López de la Teja, Sarría \& Fernández, 2017).

Hoy en día existen algunos modelos de bases de datos entre los cuales se puede mencionar: modelo de base de datos jerárquico, modelo relacional, modelo de red, modelo de base de datos orientado a objetos, modelo entidad-relación, modelo de documentos, modelo entidadatributo-valor, esquema de estrella, modelo relacional de objetos. 
Los modelos analizados son el modelo Relacional y el Entidad Relación como se observa en la tabla1.

Tabla1. Comparación Modelo Relacional - Entidad Relación

\begin{tabular}{lll}
\hline \multicolumn{1}{c}{ Criterio } & \multicolumn{1}{c}{ Relacional } & \multicolumn{1}{c}{ Entidad-Relación } \\
\hline Representación & Tablas & $\begin{array}{l}\text { Gráficos, solo representación } \\
\text { conceptual }\end{array}$ \\
Relación de la información & $\begin{array}{l}\text { Relación de presentación de } \\
\text { conexión de tablas }\end{array}$ & Relación a través de entidades \\
Tuplas & Registros de la tabla & No se ingresa información \\
Entidades & & \\
Clave & $\begin{array}{l}\text { Representa un atributo o } \\
\text { conjunto de atributos para } \\
\text { forma parte de la clave }\end{array}$ & $\begin{array}{l}\text { Solo representa una clave en la } \\
\text { entidad - no en las relaciones }\end{array}$ \\
A través de la & $\begin{array}{l}\text { estudio } \\
\text { representación de sus tuplas } \\
\text { se puede realizar } \\
\text { efectivamente que se } \\
\text { cumpla las reglas de } \\
\text { normalización. }\end{array}$ & $\begin{array}{l}\text { No se identifica inconvenientes } \\
\text { de duplicidad de información }\end{array}$ \\
\hline & $\begin{array}{l}\text { El modelo aplica SQL } \\
\text { Lenguaje de Consulta }\end{array}$ & $\begin{array}{l}\text { Es la representación más se } \\
\text { puede aplicar a cualquier SGBD }\end{array}$ \\
\hline
\end{tabular}

Fuente: Elaboración propia del autor. (2019)

El diseño de base de datos se compone de: diseño conceptual, diseño lógico y diseño físico.

El diseño conceptual es una representación del mundo real, es lo que se desea plasmar en el sistema informático, para poder automatizar los requerimientos del usuario, se necesita mucha interacción del usuario con el diseñador del sistema. 
El diseño lógico es plasmar lo elaborado en la fase de diseño conceptual a un sistema gestor de base de datos, es el estado de colocar lo bosquejado en un repositorio informático.

El diseño físico es el cómo se guarda la información y conocer el lenguaje de programación con el que se va a trabajar.

Es por ello que la metodología se basa en el procedo de un diseño conceptual y diseño lógico combinando modelos como el Entidad Relación y el Relacional.

El modelo entidad relación es una herramienta que permite representar de manera simplificada los componentes que participan en un proceso de negocio y el modo en el que estos se relacionan entre sí. (Hidalgo, 2017).

El modelo de datos relacional organiza y representa los datos en forma de tablas o relaciones. (San Miguel, 2015).

Para la realización de la metodología se propone a través de un algoritmo. Un algoritmo son pasos a seguir de forma ordenada y finita, cada paso siguiente indica lo que se debe realizar, un algoritmo siempre tendrá un Inicio y un Fin, por lo que se debe estar cien por ciento seguro que se logrará llegar a la meta a través del mismo, siempre procurando cometer la mínima cantidad de errores.

Una forma abstracta de plantear una estrategia es en la forma de un "algoritmo", es decir una secuencia de instrucciones cada una de las cuales representa una tarea bien definida y puede ser llevada a cabo en una cantidad finita de tiempo y con un numero finito de recursos computacionales. Un requerimiento fundamental' es que el algoritmo debe terminar en un numero finito de pasos, de esta manera ' el mismo puede ser usado ' como una instrucción en otro algoritmo más complejo. (Bottazzi et al., 2017)

El algoritmo a través de una metodología optimiza el tiempo para la creación de una base de datos.

Gomez y Salgado. (2016) afirman que "un algoritmo permite realizar una actividad mediante pasos sucesivos que no generen dudas a quien deba realizar dicha actividad, dados un estado inicial y una entrada, siguiendo los pasos sucesivos se llega a un estado final”.

El propósito es mejorar los tiempos de desarrollo a través de la planificación de una base de datos de calidad, hay que recordar que, si se desea desarrollar un sistema con base de datos, el puntal, el núcleo del sistema es la base de datos así se consiga un diseño de alta calidad en los formularios que manipulan la base de datos a través del sistema gestor de base de datos si la base de datos está con un mal diseño existirá un perjuicio pues implicaría volver a realizar un bosquejo de la misma con lo que implica tiempo, dinero y molestias al usuario final. 
La propuesta metodológica se centra en el diseño de la base de datos combinando con los dos modelos escogidos, mencionando así las siguientes fases:

Fase conceptual. (Qué representar): Será la iteración del cliente con el desarrollador, en esta fase debe existir total comunicación entre ambos actores. La información que se recoge son las formas de trabajo del cliente para poder plasmar en un modelo Entidad - Relación el cual ayuda a través de sus gráficos la representación del mundo real a un entendimiento informático.

Al realizar el bosquejo del diseño a través de conceptos planteados por el usuario no significa que ese primer intento de plasmar gráficamente el mecanismo de trabajo de la empresa será el definitivo, van a existir algunas entrevistas cliente - diseñador hasta que ambos estén cien por ciento convencidos de lo que desea informatizar de parte del cliente y lo que entendió el diseñador de los requerimientos del cliente.

Una vez realizada la fase de entrevista diseñador - usuario se debe realizar la fase de análisis por parte del diseñador de la base de datos, identificando atributos conocidos como características del objeto a representar, relaciones entre entidades y su cardinalidad. Entendiéndose por cardinalidad a la forma en que se relacionan el o las entidades; como parte de la fase se deben identificar claves primarias tratando de que se cumpla que se identifique de manera única un objeto dentro de la entidad.

Al expresar un diseño en modelo de Entidad-Relación no se verifica que se cumpla la no duplicidad de información pues una relación puede transformarse según sea el caso en una tabla en el Sistemas Gestor de Base de Datos seleccionado, por lo tanto, una representación gráfica en el modelo Entidad - Relación y es por esto que se debe pasar a un modelo que ayude a interpretar de mejor manera la base de datos e identificar las posibles anomalías del mismo.

Se recomienda que una vez realizado todos los pasos mencionados se proceda a realizar una vez más una jornada de trabajo diseñador-cliente para explicar al cliente lo que el diseñador interpretó de las primeras fases de trabajo cliente-diseñador, si existe algún cambio se deberá volver a desarrollar la Fase Conceptual hasta llegar a una satisfacción de los requerimientos por parte del cliente y así poder pasar a una Fase Lógica.

Cada fase debe ser documentada, la documentación permite la seguridad y seriedad en el trabajo realizado, pues debe realizarse un acta donde cliente-diseñador están totalmente de acuerdo en lo que va a representar en la siguiente fase de trabajo y si se realiza en la empresa un cambio de personal, el siguiente diseñador al mando tendrá toda la información para continuar con el desarrollo dela Base de Datos y de esta forma no exista prejuicio con el cliente como con la empresa desarrolladora. 
Fase lógica (Analizar ambiguiedades): El modelo a emplear en la presente fase es el modelo Relacional, como ya se indicó es un modelo que se basa en tablas y estas tablas deben estar relacionadas, el utilizar el modelo ayuda a tener una mejor visión de cómo están relacionados los datos, si se cumple las reglas del negocio proporcionada por el cliente.

Las entidades pasar a ser tablas y las relaciones del tipo de muchos a muchos se convierten en tablas, al trabajar con tablas relacionadas se puede agregar información conocidas como registros o tuplas y se puede verificar que la duplicidad de información no exista, si es el caso se puede pasar atributos a formar parte de la clave primaria y así poder solucionar la duplicidad de información, además el modelo aplica la normalización.

La normalización es el proceso de simplificar la relación entre los campos de un registro. Por medio de la normalización un conjunto de datos en un registro se reemplaza por varios registros que son más simples y predecibles y, por lo tanto, más manejables. (Trueba, 2016)

Es recomendable en el diseño de una base de datos indagar por lo menos hasta la tercera forma normal, de esta manera no se asegura un total control de redundancia de datos, pero por lo menos se puede manipular la información de manera controlada. Otro asunto muy importante es la seguridad que se debe dar al acceso de la Base de Datos este proceso ya corresponde totalmente al diseñador, pero se considera que la fase lógica es la más indicada por la etapa de diseño para limpieza del esquema.

Ya en este proceso se puede agregar información a las tablas con lo que se puede involucrar al cliente para reafirmar lo planteado hasta el momento y verificar los informes que desea obtener del mismo, pues al trabajar con datos es más fácil para el cliente identificar lo que se va a informatizar, lo que se realiza en la fase es pulir la conceptualización antes de proceder a un medio informático.

No se debe pasar por alto la documentación, al tener una fase más que culmina con una pulida al desarrollo de conceptos es muy importante documentar y firmar acuerdos. Hasta esta fase es muy importante involucrar al cliente en el desarrollo de la metodología.

Fase física (Repositorio Informático): Lo que se requiere en esta fase es la representación de la información del diseño gráfico al esquema del Sistema Gestor de Base de Datos escogido, una vez establecidos las reglas de negocio se procederá a implementar en una forma real e informática verificar que las mismas se cumplan, se debe recordar que lo que se plasmará en cómo trabaja la empresa más no como se desea que trabaje, pues las reglas del negocio las proporciona el cliente.

Un Sistema Gestor de Base de Datos (SGBD) o DataBase Managenent System (DBMS) es un sistema que permite la creación, gestión y administración de bases de datos, así como la elección y manejo de las estructuras necesarias para el 
almacenamiento y búsqueda de información del modo más eficiente posible.(Marin, 2019)

Se debe tomar en cuenta las transacciones y consultas que se necesite realizar en la base de datos, espacio en disco, tomando en cuenta el crecimiento vertical de la misma.

La escalabilidad vertical o hacia arriba, este es el más simple, pues significa crecer el hardware de uno de los nodos, es decir aumentar el hardware por uno más potente, como disco duro, memoria, procesador, etc. pero también puede ser la migración completa del hardware por uno más potente. El esfuerzo de este crecimiento es mínimo, pues no tiene repercusiones en el software, ya que solo será respaldar y migrar los sistemas al nuevo hardware. (Blancarte, 2017).

Para culminar la fase no se puede pasar por alto la documentación, siempre es significativo documentar y firmar acuerdos. El cliente siempre tiene que estar involucrado en cada fase del diseño, hay que recordar que en esta fase se confirma la adquisición de requisitos como hardware y SGBD lo cual se debe conocer de parte del diseñador una lista de posibilidades de costos y facilidad de adquisición, así como niveles de seguridad, donde el cliente decidirá lo que puede o no cubrir.

\section{Resultados.}

Una vez explicada cada fase de diseño se puede realizar el algoritmo con el cual como todo algoritmo su objetivo es cumplir una solución (meta) a través de una secuencia de pasos ordenada.

\section{Algoritmo de Diseño de la Base de Datos.}

1. Inicio

2. Cliente solicita informatizar su trabajo diario

3. Cliente comunica necesidad al diseñar

4. Cliente explica la forma de trabajar de la empresa

5. Diseñador recoge información

6. Diseñador realiza bosquejo en el Modelo Entidad - Relación

7. Diseñador presenta bosquejo al cliente

8. Si el cliente está de acuerdo va a al paso 9 caso contrario se vuelve al paso 4

9. El Diseñador empieza la fase de análisis:
a) Identifica entidades
b) Atributos
c) Relaciones
d) Cardinalidades
e) Claves primarias
10. Diseñador presenta bosquejo al cliente 
11. Si el cliente está de acuerdo va al paso 12 caso contrario se vuelve al paso 4

12. Realizar documentación y acta

13. Diseñar Modelo Relacional

14. Pasar entidades a tablas identificando claves primarias y claves foráneas

15. Identificar las relaciones que deben transformarse en tablas junto con sus claves

16. Verificar a través de registros las tuplas duplicadas y ambigüedad de la información.

17. Verificar si se debe aplicar la $1 \mathrm{FN}$ si es si pasar al paso 18 caso contrario ir al paso 19

18. Aplicar la 1FN

19. Verificar si se debe aplicar la $2 \mathrm{FN}$ si es si ir al paso 19 caso contrario ir al paso 20

20. Aplicar la 2FN

21. Verificar si se debe aplicar la $3 F N$ si es si ir al paso 21 caso contrario ir al paso 22

22. Aplicar la 3FN

23. Crear tablas necesarias para ejecutar controles de seguridad del sistema.

24. Diseñador presenta modelo al cliente

25. Si el cliente está de acuerdo va al paso al paso 26 caso contrario se vuelve al paso 13

26. Realizar documentación y acta

27. Plasmar el modelo Relacional al esquema Relacional del SGBD escogido

28. Identificar transacciones y consultas

29. Si el cliente está de acuerdo continuar con el paso 30 caso contrario se vuelve al paso 28

30. Realizar documentación y acta

31. Fin

Qué tan eficiente es el algoritmo, para esto la base será el tiempo de ejecución, cada paso toma el valor de 1, de igual forma las preguntas lógicas tendrán el valor de 1.

La condición suele ser de $\mathrm{O}(1)$, complejidad a sumar con la peor posible, bien en la rama THEN, o bien en la rama ELSE. En decisiones múltiples (ELSIF, CASE), se tomará la peor de las ramas. (Mañas, 2017) (Sic)

El algoritmo es independiente de la máquina y el lenguaje de programación, y el SGBD, los pasos son claros y concretos. Es finito el número de pasos, es preciso cada paso realiza algo concreto, devuelve información de salida. 


\section{Análisis del Algoritmo.}

Tabla2. Evaluación de la complejidad del Algoritmo

\begin{tabular}{lc}
\hline \multicolumn{1}{c}{ PROCESO } & PESO \\
\hline Inicio & 1 \\
Cliente solicita informatizar su trabajo diario & 1 \\
Cliente comunica necesidad al diseñar & 1 \\
Cliente explica la forma de trabajar de la empresa & 1 \\
Diseñador recoge información & 1 \\
Diseñador realiza bosquejo en el Modelo Entidad - Relación & 1 \\
Diseñador presenta bosquejo al cliente & 1 \\
Si el cliente está de acuerdo va a al paso 9 caso contrario se vuelve al paso 4 & 1 \\
El Diseñador empieza la fase de análisis: & 1 \\
Diseñador presenta bosquejo al cliente & 1 \\
Si el cliente está de acuerdo va al paso 12 caso contrario se vuelve al paso 4 & 1 \\
Realizar documentación y acta & 1 \\
Diseñar Modelo Relacional & 1 \\
Pasar entidades a tablas identificando claves primarias y claves foráneas & 1 \\
Identificar las relaciones que deben transformarse en tablas junto con sus & 1 \\
Claves & 1 \\
Verificar a través de registros las filas duplicadas y ambigüedad de la & 1 \\
información. & 1 \\
Verificar si se debe aplicar la 1FN si es si pasar al paso 17 caso contrario ir al & 1 \\
paso 18 & 1 \\
Aplicar la 1FN & 1 \\
Verificar si se debe aplicar la 2FN si es si ir al paso 19 caso contrario ir al paso & 1 \\
An & 1 \\
Aplicar la 2FN & 1 \\
Verificar si se debe aplicar la 3FN si es si ir al paso 21 caso contrario ir al paso & 1 \\
Aplicar la 3FN & 1 \\
Crear tablas necesarias para ejecutar controles de seguridad del sistema. & 1 \\
Diseñador presenta modelo al cliente & 1 \\
Si el cliente está de acuerdo va al paso 26 caso contrario se vuelve al paso 13 & 1 \\
Realizar documentación y acta & 1 \\
Plasmar el modelo Relacional al esquema Relacional del SGBD escogido & 1 \\
Identificar transacciones y consultas & 1 \\
Si el cliente está de acuerdo continuar con el paso 30 caso contrario se vuelve & 1 \\
al paso 28 & 1 \\
Fealizar documentación y acta & 1 \\
Orden de la función O(1) & 1 \\
\hline & 1 \\
\hline
\end{tabular}

Fuente: Elaboración propia del autor. (2019)

Es un algoritmo del tipo constante $\mathrm{O}(1)$ es decir una complejidad constante o tiempo constante, por lo tanto, es un algoritmo que no será afectado en su rendimiento debido a la cantidad de datos que se maneje.

A partir del análisis de complejidad se puede exponer que el algoritmo a través de una metodología optimiza el tiempo para la creación de una base de datos. 
Otra forma de representar un algoritmo y mejor apreciación es el algoritmo gráfico conocido como diagrama de flujo, entonces se procede para su mejor apreciación el algoritmo propuesto es narrativo (Lenguaje natural) se lo representa a través de gráficos (Diagrama de flujo) en la siguiente imagen, aplicando Microsoft Visio 2016.

Microsoft Visio (Microsoft, 2016), está diseñado para personas que buscan una poderosa plataforma de diagramación con una rica serie de galerías de símbolos integradas. Ayuda a los usuarios a simplificar información compleja por medio de diagramas sencillos y fáciles de comprender. Visio Standard incluye galerías de símbolos para diagramas empresariales, diagramas básicos de red, organigramas, diagramas de flujo básicos y diagramas generales de usos múltiples. (Jiménez et al., 2016)

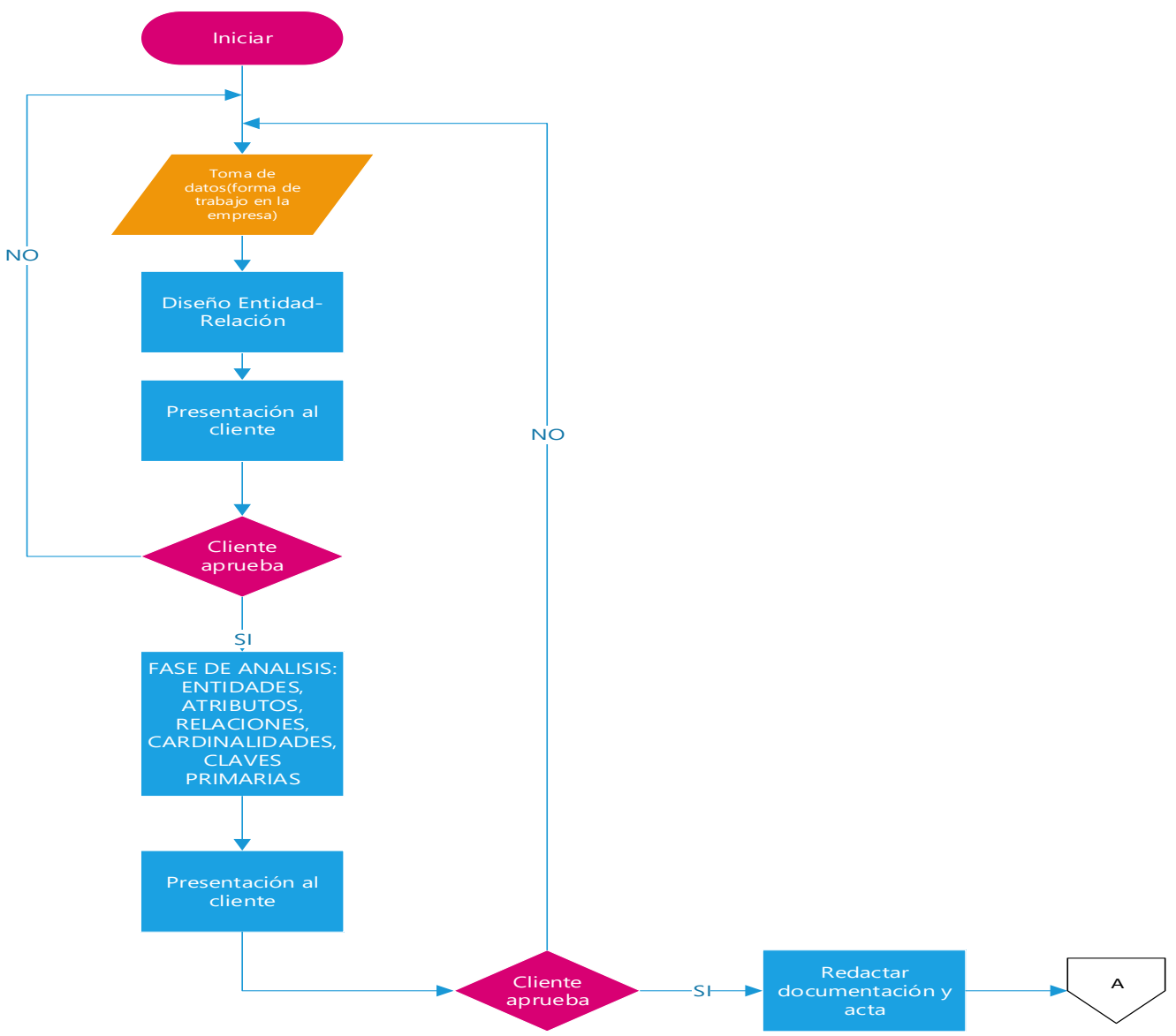

Figura 1. Diagrama de flujo del algoritmo.

Fuente: Elaboración propia del autor en programa Visio. (2019) 


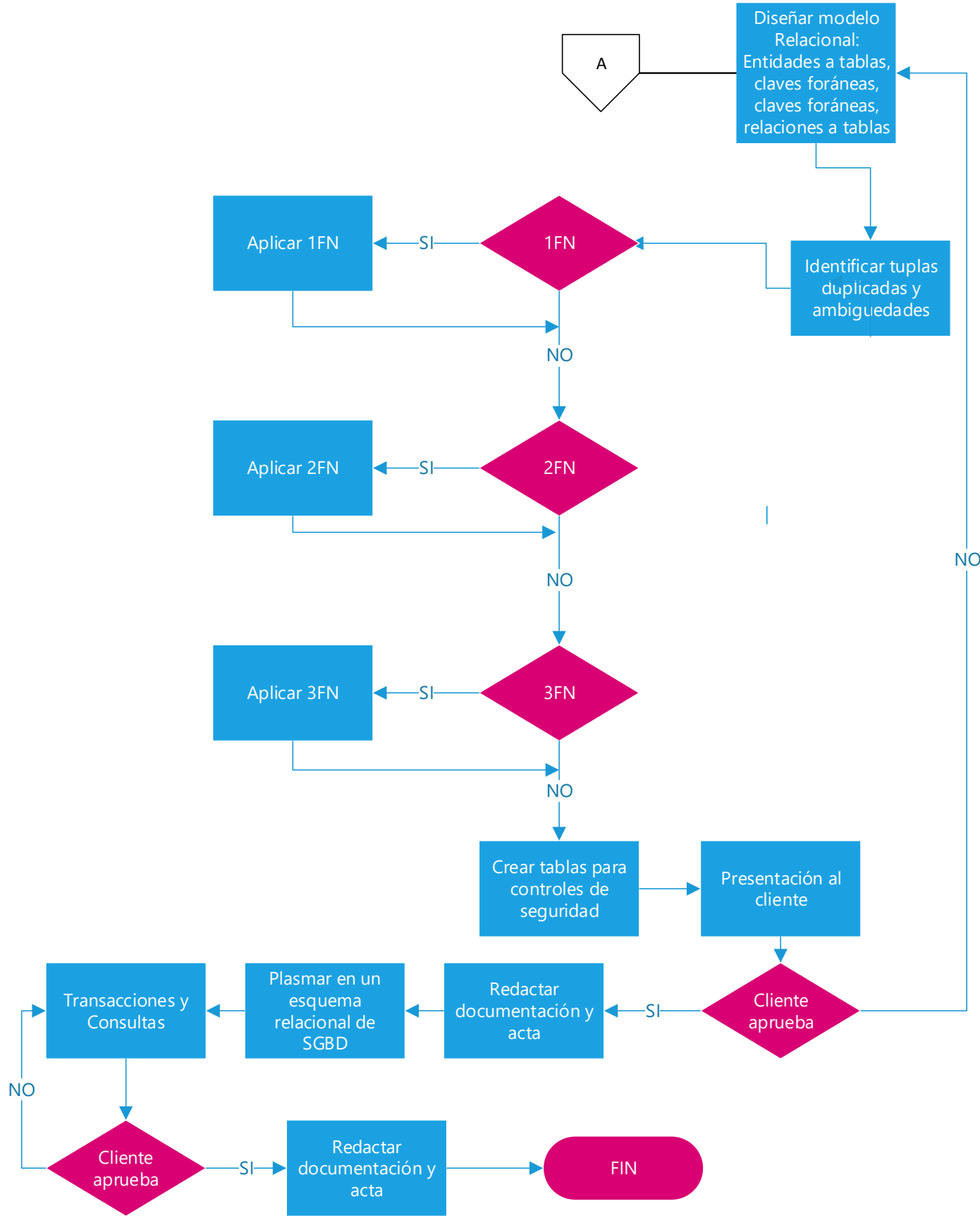

Figura 2. Diagrama de flujo del algoritmo.

Fuente: Elaboración propia del autor en programa Visio. (2019)

Para el algoritmo es muy importante la intervención del cliente para poder alcanzar la meta en cada fase siempre se verifica la aprobación del cliente, el cliente es considerando parte del equipo durante la fase de diseño. 


\section{Conclusiones.}

- El representar una metodología a través de un algoritmo facilita el diseño de un esquema, un algoritmo se basa en pasos con el fin de lograr la meta de solución con pasos finitos, secuenciales o de confirmación consiguiendo el avance de cada paso con la seguridad que el paso anterior fue completado en su totalidad.

- Al finalizar cada fase es importante involucrar al cliente/usuario pues debe estar cien por ciento planteado el mundo real en el diseño del esquema de la Base de Datos pues es en base a este esquema que el desarrollador basará la programación de presentación y ejecución final del software.

- La metodología planteada es en base a las fases de diseño de bases de datos aplicado con los modelos Entidad-Relación y Modelo Relacional utilizando las teorías de metodologías de ingeniería de software donde es muy importante la participación del cliente. El algoritmo es una secuencia con instrucciones de preguntas para continuar el mismo caso contrario repetirá pasos necesarios para continuar, la solución del algoritmo es la creación de la Base de Datos lista para ser implementada en cualquier SGBD. Además, la utilización de un modelo Entidad-Relación y a su vez pasar a un modelo Relacional permite que la no interrupción de la secuencia se esté seguro de un diseño conciso en base al requerimiento del cliente, debido a que el cliente es una pieza clave para el diseño del mismo.

\section{Referencias Bibliográficas.}

Blancarte, Oscar.(2017).Escalabilidad Vertical y Horizontal. Recuperado de: https://www.oscarblancarteblog.com/2017/03/07/escalabilidad-horizontal-y-vertical

Bottazzi, C., Costarelli, S.,Dalcin, L., Galizzi, D., Gimenez, J., Olivera, J., ...Storti, M. (2017). Algoritmos y Estructuras de Datos. Recuperado de: https://cimec.org.ar/ mstorti/aed/aednotes.pdf

Codina, Luis (2017). Estructura y funciones de una base de datos académica. Recuperado de: https://www.lluiscodina.com/bases-de-datos-academicas-registros/

Garcia, Francisco.(2018). Ingeniería de Software. Salamanca. Recuperado de: https://repositorio.grial.eu/bitstream/grial/1228/1/07-rep.pdf

Gomez,E., Salgado,N.(2016). Logica de Algoritmos- Un enfoque Practico- Programación en Lenguaje C. Recuperado de: https://www.researchgate. net/publication /2966719 28Logica_de_Algoritmos-_Un_enfoque_Practico-_Programacion_en_Lenguaje_C

Hidalgo, Luis.(2017). Modelo entidad relación: descripción y aplicaciones. Recuperado de: https://www.icemd.com/digital-knowledge/articulos/modelo-entidad-relacion-descr ipcion-aplicaciones/ 
Jimenez, M., De la Hoz, S., Huyke, A., Mendoza, M., Rangel, E., Pastrana J., Castro, L., Ospino, F. (2016). Software para la elaboración de diagramas de estudio del trabajo como herramienta facilitadora en el proceso de enseñanza - Aprendizaje de métodos y tiempos en las actividades productivas: Diagramet. Espacios, Vol.38(N020). Recuperado de: https://www.saberespractico.com/investigacion/ap a/como-citar-unarticulo-de-revista-segun-la-normativa-apa/

López de la Teja, L. J., Sarría Stuart Á., \& Fernández Álvarez, D. (2017). La formación de conceptos en el proceso de enseñanza-aprendizaje de los sistemas de gestión de bases de datos. Revista Conrado, 13(57), 139-145. Recuperado de http://conrado.ucf.edu.cu/index.php/conrado

Mañas, José. (2017). Análisis de Algoritmos - Complejidad. Recuperado de: http://www.dit.upm.es/ pepe/doc/adsw/tema1/Complejidad.pdf

Marin, Rafael. (2019). Los gestores de bases de datos más usados en la actualidad. Recuperado de: https://revistadigital.inesem.es/informatica-y-tics/los-gestores-debases-de-datos-mas-usados/

Merchán, Oswaldo. (2016). Diseño de Base de Datos. Recuperado de: http://publicaciones.uazuay.edu.ec/index.php/ceuazuay/catalog/view/5/5/37-2

Rivas, C., Corona, V., Gutierrez, J., Hernandez, L. (2015). Metodologías actuales de desarrollo de software. Recuperado de: http://www.ecorfan.org /bolivia/resear chjournalsTecnologia_e_innovacion/vol2num5/Tecnologia_e_Innovacion_Vol2_Nu m5_6.pdf

San Miguel, Gonzalo. (2015). Diseño e Implementación de una Herramienta Visual que Obtenga el Modelo Relacional Lógico a partir de un Diagrama Entidad Relación. Recuperado de: https://ebuah.uah.es/dspace/bitstream/handle/10017/28479/TFGSan\%20Miguel-Oteo-2016.pdf?sequence=1\&isAllowed $=y$

Trueba, Adrian. (2016). Proceso de normalización de base de datos en una modelo relacional. Recuperado de: https://core.ac.uk/download/pdf/80532387.pdf

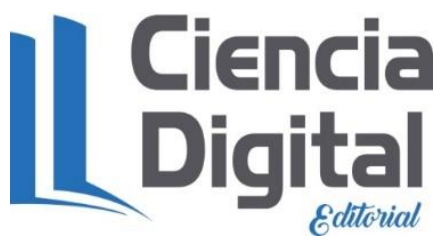




\section{PARA CITAR EL ARTÍCULO INDEXADO.}

Valverde González, V. L. (2019). Algoritmo para el diseño de base de datos. Ciencia Digital, 3(3.2.1), 5-19. https://doi.org/10.33262/cienciadigital.v3i3.2.1.778

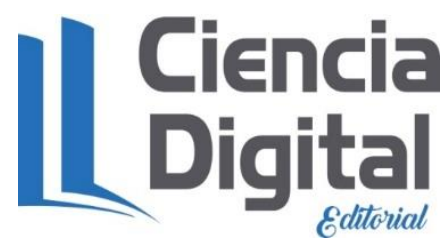

El artículo que se publica es de exclusiva responsabilidad de los autores y no necesariamente reflejan el pensamiento de la Revista Ciencia Digital.

El artículo queda en propiedad de la revista y, por tanto, su publicación parcial y/o total en otro medio tiene que ser autorizado por el director de la Revista Ciencia Digital.
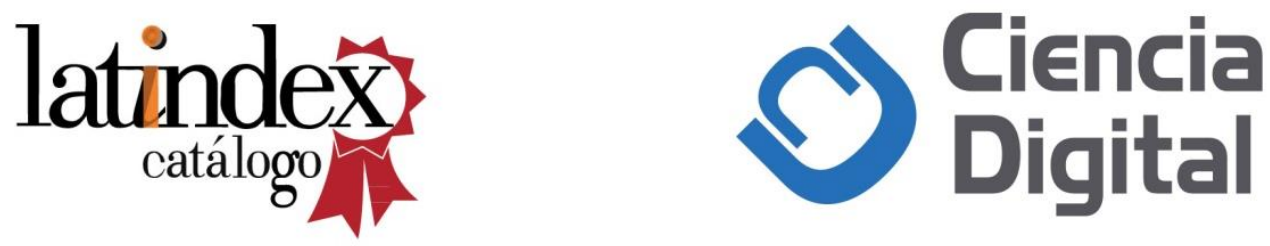